\title{
DISTROFIA MIOTÓNICA TIPO 1: REPORTE DE UN CASO DE UN PACIENTE COLOMBIANO
}

\author{
Diana Castro- $\mathrm{R}^{1}$ \\ Julián Ramírez-C² \\ Wilmar Saldarriaga ${ }^{3}$ \\ Carolina Isaza ${ }^{4}$
}

\section{RESUMEN}

Introducción: La distrofia miotónica es una enfermedad poco frecuente de origen genético. Se produce por aumento de repeticiones de la tripleta CTG en el gen DMPK (locus 19q13.32), o por aumento de repeticiones de CCTG en el gen ZNF9 (locus3q21.3). Su fenotipo es variable y sus principales características son la debilidad muscular progresiva y la miotonía.

El objetivo de esta publicación es reportar un caso colombiano de distrofia miotónica tipo 1 con diagnóstico molecular y contribuir a la construcción de datos epidemiológicos locales sobre esta patología. Además, aportar información a médicos generales, pediatras, internistas, fisiatras, neurólogos, y en general al personal de salud que puede tener contacto con pacientes con debilidad muscular progresiva, escenario en el cual la distrofia miotónica es una posibilidad diagnóstica a considerar.
Descripción del caso: Hombre de 37 años, con historia de pobre succión neonatal, retraso en los hitos del desarrollo, discapacidad intelectual y, en la adolescencia, aparición de debilidad progresiva generalizada, miotonía y disfagia. El Southernblot y PCR del gen DMPK mostraron un alelo expandido en un rango entre 1100 a 1700 repeticiones del triplete CGT y un alelo normal, confirmando el diagnóstico de distrofia miotónicatipo 1.

Conclusión: El paciente aquí reportado presentó fenotipo sugestivo de DM1; el diagnóstico fue confirmado con la prueba molecular. Con el resultado fue posible realizar una consejería genética adecuada y brindar información sobre la enfermedad.

Palabras clave: Distrofia miotónica, enfermedades genéticas congénitas, expansión de repetición de trinucleótido, enfermedades raras, pruebas genéticas.

\footnotetext{
1 MD., Universidad del Valle. Correo electrónico: dicar9001@hotmail.com

${ }^{2}$ MD. MSc. Profesor Departamento de Morfología-Escuela de Ciencias Básicas-Facultad de Salud-Universidad del Valle. Correo electrónico: juracheyne@gmail.com

${ }_{3}^{3}$ MD. MSc. Profesor Departamento de Morfología-Escuela de Ciencias Básicas-Facultad de Salud-Universidad del Valle. Correo electrónico: wilmar.saldarriaga@correounivalle.edu.co

4 MD. MSc. Profesora Departamento de Morfología-Escuela de Ciencias Básicas-Facultad de Salud-Universidad del Valle. Correo electrónico: carolina.isaza@correounivalle.eu.co
} 


\section{MYOTONIC DYSTROPHY TYPE 1: CASE REPORT OF A COLOMBIAN PATIENT}

\section{ABSTRACT}

Introduction: Myotonic dystrophy (MD) is a rare genetic disease. It is produced by an increased repetition of the CTG triplet in the DMPK gene (locus 19q13.32), or by increasing repetitions of CCTG in the ZNF9 gene (locus 3q21.3). Its phenotype is variable, and its key features are progressive muscle weakness and myotonia.

The aim of this publication is to report a Colombian case of myotonic dystrophy type 1 with molecular diagnosis and to contribute to the construction of local epidemiological data on this pathology. Also, to provide information to general practitioners, pediatricians, internists, physiatrists, neurologists, and health personnel who may have contact with patients with progressive muscle weakness, scenario in which

\section{INTRODUCCIÓN}

La distrofia miotónica (DM) es una enfermedad de origen genético, con herencia autosómica dominante, que se presenta en 12,5 de cada 100000 habitantes. Se produce por aumento del número de repeticiones de la tripleta CTGenelgenDMPK (dystrophia myotonica proteinkinase) con locus 19q13.32, o por aumento de repeticiones CCTG en el gen ZNF9 (zinc finger protein) con locus 3q21.3. La enfermedad se manifiesta cuando, en alguna de las copias de uno de los genes implicados, se sobrepasa un determinado umbral de repeticiones. Es una entidad crónica, lentamente progresiva y con un espectro clínico ampliamente variable (1).

La expresión fenotípica va desde adultos asintomáticos hasta manifestaciones graves in utero. El rango abarca debilidad y pérdida de masa muscular, miotonía, cataratas, myotonic dystrophy is a diagnostic possibility to be considered. Case description: Thirty-seven year old male with a history of poor neonatal suction, delay in developmental milestones, intellectual disability and, in adolescence, the onset of progressive generalized weakness, myotonia and dysphagia. Southern blot and PCR of DMPK gene showed one expanded allele in a range between 1100-1700 repetitions of the CGT triplet and one normal allele, confirming the diagnosis of myotonic dystrophy type 1. Conclusion: The patient reported here presented a phenotype suggestive of myotonic dystrophy type 1 ; the diagnosis was confirmed by molecular testing. This result made it possible to offer a proper genetic counseling and provide information about the disease.

Key words: Myotonic dystrophy, congenital genetic diseases, trinucleotide repeat expansion, rare diseases, genetic testing.

cardiopatía, arritmias cardiacas, disfunciones endocrinológicas, cognitivas, gastrointestinales y respiratorias; complicaciones en el embarazo como aborto recurrente e inconvenientes con el uso de anestésicos (2). El fenómeno de anticipación observado en DM, que produce que las generaciones siguientes presenten un cuadro clínico de inicio cada vez más temprano y por tanto más grave, es debido a la expansión del número de repeticiones durante la gametogénesis (3).

El diagnóstico puede ser difícil debido al amplio rango de manifestaciones clínicas con diferente grado de severidad y al fenómeno de anticipación (4). Ante la sospecha clínica para confirmar el diagnóstico se deben utilizar pruebas moleculares como la reacción en cadena de la polimerasa (PCR), la PCR utilizando triple cebador (PT-PCR) o Southernblot (5). 
El objetivo de esta publicación es reportar un caso colombiano de DM1 con diagnóstico molecular a la literatura y contribuir a la construcción de datos epidemiológicos locales sobre una enfermedad genética poco frecuente. Además, aportar información a médicos generales, pediatras, internistas, fisiatras, neurólogos y al personal de salud que puede tener contacto con pacientes con debilidad muscular progresiva, escenario en el cual la DM es una posibilidad diagnóstica a considerar.

\section{MATERIALES Y MÉTODOS}

\section{Descripción del caso:}

Paciente de 37 años de edad, procedente de Cali, remitido por neurólogo al servicio de genética médica por cuadro de debilidad muscular lentamente progresiva. A los 16 años el paciente inicia con disminución de la fuerza distal notada al caérsele objetos y tropezar frecuentemente. Posteriormente, dificultad para subir y bajar escaleras, levantar objetos, desplazarse trayectos largos, en los últimos 6 meses dificultad para la deglución. También refirió diarrea crónica.

A la consulta traía electrocardiograma y electromiografía sin alteración. Enzima CreatininKinasa de $300 \mu \mathrm{mol} / 1$.

Dentro de los antecedentes personales se encontró pobre succión en el periodo neonatal que le impidió lactancia materna, no gateó, pronunció sílabas a los 2 años, caminó a los 3 años. Además, no logró terminar el bachillerato por problemas académicos. En los antecedentes familiares se encontró que 8 parientes del lado paterno de la familia, incluido el padre, tuvieron un cuadro de debilidad muscular progresiva (Figura 1).

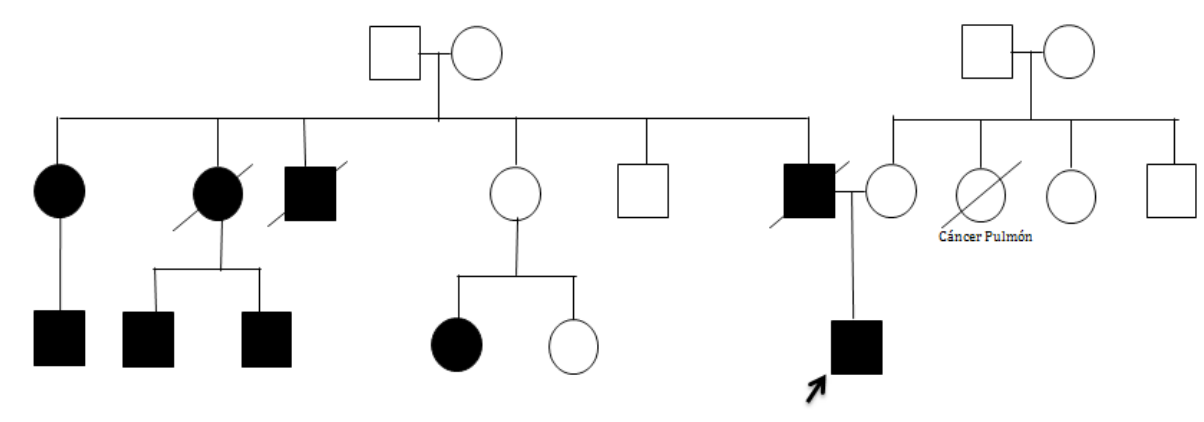

Figura 1: Heredograma de la familia reportada con DM1. Muestra que hay hombres y mujeres afectados en dos generaciones sucesivas, sugiriendo esto un patrón de herencia autosómico dominante. Alguno de los individuos de la primera generación de la línea paterna de la familia del caso índice, debió ser portador de la premutación (35-49 repeticiones de CTG del gen DMPK) o de la mutación asociada al fenotipo leve (50-150) no identificado en la memoria familiar.

En el examen físico se evidenció contextura delgada, IMC 19, cara alargada, disminución de la masa muscular temporal y facial. La fuerza muscular se encontró disminuida en las cuatro extremidades: en los músculos distales $3 / 5$ y en los proximales $4 / 5$; además, presentó dificultad para relajar la mano después de empuñarla con fuerza (miotonía).

En el análisis clínico se consideró que el paciente presentó manifestaciones desde el periodo neonatal (pobre succión) y retraso en los hitos del 
desarrollo; sin embargo, logró caminar y llevar una vida relativamente normal en el aspecto motriz; posteriormente, en la adolescencia, tuvo disminución progresiva de la fuerza. Con esta historia y los antecedentes familiares sugestivos de trastorno de debilidad muscular progresiva de herencia autosómica dominante, se hizo una impresión diagnóstica de DM1 y se solicitó prueba molecular para cuantificación de tripletas CTG en el gen DMPK. Se tomaron 8 centímetros cúbicos de sangre en dos tubos con anticoagulante EDTA y se enviaron en frío al Laboratorio de Genética Médica de BMC (Baylor College of Medicine), donde a través de Southern (de DNA digerido con Ncol y BamHI) y PRC (Polymerase Chain Reaction) se determinó el tamaño de la región de repeticiones CTG del gen DMPK.

El resultado mostró que el paciente tenía un alelo del gen DMPK expandido en un rango entre 1100 a 1700 repeticiones del triplete CGT y un alelo normal, confirmando el diagnóstico de DM1.

Se le realizó consejería genética explicando que, debido a alteración en el gen DMKP, él y sus familiares afectados tendrían un $50 \%$ de riesgo de tener hijos con síntomas similares o más pronunciados que los de él. Además, se explicó sobre la no conveniencia del uso de estatinas y sobre su riesgo incrementado de reacciones adversar a sedantes, opioides y anestésicos. Se explicó que debería realizarse anualmente un Holter, curva de tolerancia a la glucosa, TSH y T4, valoración por oftalmólogo.

Se remitió a psicología, fisiatría y neurología, quienes ordenaron terapia física encaminada a fortalecer el tono muscular y psicoterapia para facilitar la aceptación de la enfermedad. El paciente tuvo una pobre respuesta a las terapias y presentó crisis depresivas que necesitaron tratamiento farmacológico por psiquiatra.

La valoración por oftalmólogo no encontró cataratas ni otras patologías oculares. La curva de tolerancia a la glucosa, el TSH y T4 no mostraron valores alterados.

\section{DISCUSIÓN}

La distrofia miotónica es una rara enfermedad genética, de la que existen los tipos 1 y 2, los cuales son causados por mutaciones en los genes DMPK y ZNF9 respectivamente. Si bien los dos tipos coinciden en la debilidad muscular progresiva y en que el patrón de herencia es autosómico dominante, la presentación clínica es diferente. Una comparación entre DM1 y DM2 puede verse en la tabla 1, el paciente aquí reportado tenía hallazgos clínicos sugestivos de la tipo 1, lo cual justificó la prueba molecular para el gen específico (1).

Tabla 1: Comparación entre distrofia miotónica 1 y 2

\begin{tabular}{|c|c|}
\hline Distrofia miotónica 1 & Distrofia miotónica 2 \\
\hline $\begin{array}{l}\text { Inicio desde antes del primer año } \\
\text { hasta la adultez }\end{array}$ & Inicio entre los 8 y 60 años \\
\hline & \\
\hline Pre & \\
\hline e la debilidad: distal a & de la debilidad: proximal \\
\hline $\begin{array}{l}\text { Causa: expansión de más de } 50 \\
\text { repeticiones CTG en el gen DMPK }\end{array}$ & $\begin{array}{l}\text { Causa: expansión de más de } 5000 \\
\text { repeticiones CCTG en el gen ZNF9 }\end{array}$ \\
\hline
\end{tabular}


DMPK es un gen de $14 \mathrm{~kb}$ que codifica una proteína de 624 aminoácidos, la cual es una kinasa de serina-treonina dependiente de cAMP (6) y tiene funciones específicas en la excitabilidad muscular. El defecto en este gen, relacionado con la DM1, es el número anormal de la tripleta CTG en una región no transcrita del extremo 3 prima. El número de repeticiones en la población normal varía entre 5 y 34 veces; los individuos con número de repeticiones entre 35 y 50 se consideran en premutación, no tienen síntomas o estos son muy leves, pero pueden expandir a su progenie; entre 50 y 150 repeticiones la afectación es leve, se encuentran cataratas y miotonía leve; en el rango entre 100 y 1000 repeticiones se presentan los signos clásicos de DM1, es decir, debilidad muscular, miotonía, cataratas, alopecia, arritmias, entre otros; y cuando el número de repeticiones es mayor de 1000 se produce una distrofia miotónica congénita con hipotonía neonatal e infantil, déficit respiratorio, discapacidad intelectual y signos de la forma clásica (7).

Esta enfermedad presenta el fenómeno de anticipación genética, produciéndose un aumento del número de tripletas en la progenie según el sexo del padre que lo transmite. Los hombres (padres) expanden a su progenie cuando tienen alelos con rango entre $40 \mathrm{y}$ 80 repeticiones, mientras que las mujeres (madres) expanden si tienen alelos con más de 80 tripletas $(6,8)$. El fenómeno de constricción, disminución del número de tripletas se ha visto hasta en el 5\% de los casos(9).

Los pacientes con DM1 son heterocigotos para la expansión de la repetición CTG en DMPK, por tanto la herencia es dominante $(10,11)$. En el caso aquí reportado, el rango de repetición de la tripleta en el alelo anormal estuvo entre 1100 y 1700 explicando esto los síntomas del paciente, consistentes en pobre succión neonatal, retraso en los hitos del desarrollo, discapacidad intelectual y en la adolescencia aparición de signos de la forma clásica de DM1 como miotonía, debilidad progresiva generalizada, trastornos de la deglución y pobre respuesta al tratamiento.
La expansión del gen DMPK tiene consecuencias fisiopatológicas que pueden correlacionarse con las características clínicas de la enfermedad:

Alteración de la expresión de DMPK y otros genes vecinos: La expansión de repeticiones CTG interfiere con la configuración local de la cromatina, lo cual afecta la expresión de DMPK y otros genes vecinos como: DMAHP (dystrophia myotonica associated home domain protein)/ SIX5, DMWD (dystrophia myotonica containing WD) y MBNL1 (muscle blind protein) $(12,13)$.

Relacionadas con el fenotipo muscular: Normalmente, la DMPK fosforila los canales de $\mathrm{Na}^{+}$dependientes de voltaje específicos del músculo y regula la excitabilidad de las células musculares. Al estar alterada la expresión de esta proteína, también se altera dicha regulación (14). Por otro lado, en condiciones normales, el transcrito DMPK es procesado en el núcleo, luego es transportado al citoplasma donde se une a proteínas específicas como: CUG-BP o CELF1 y finalmente es traducido. En los pacientes con DM1, los transcritos del alelo mutante y del alelo normal son retenidos en el núcleo porque las repeticiones CUG secuestran proteínas transportadoras de mRNA. Los transcritos de DMPK tienen un tracto largo de CUG que forman estructuras tipo horquilla (15) y se observan agregados de transcritos DMPK en los focos ribonucleicos en el núcleo. Los agregados de repeticiones CUG en el núcleo secuestran la proteína MBNL1, cuyo gen está localizado en el cromosoma 3, y esta alteración es probablemente la etiología de los efectos fenotípicos como la miotonía y otros síntomas a nivel de sistema musculoesquelético de la $\operatorname{DM} 1(16,17,18)$.

Relacionadas con cataratas: el silenciamiento de los genes DMAHP/SIX5 secundario a la expansión de CTG en DMPK, se ha relacionado con la formación de cataratas (12).

Relacionadas con otras características fenotípicas: otro evento celular que se observa es el aumento 
de CUGBP1. Tanto CUGBP1como MBNL1 regulan el corte y empalme (splicing) de mRNA (s) que codifican proteínas como la del canal de cloro 1, el receptor de insulina y la troponina $\mathrm{T}$ (19). El splicing alterado de estos mRNA(s) afecta la producción de las correspondientes proteínas, lo que puede explicar la naturaleza multisistémica de las manifestaciones de $\operatorname{DM} 1(17,18,19,20,21)$.

La gravedad y la edad de aparición de los síntomas en los pacientes con DM1 se correlaciona con el número de repeticiones. Los casos con 50 a 150 repeticiones tienen manifestaciones leves en la sexta década de la vida o después; los casos clásicos se manifiestan a temprana edad y tienen entre 150 y 1000 repeticiones; y la tercera variedad es la congénita, que está asociada a polihidramnios, contracturas articulares e hipotonía al nacer; y son casos que tienen más de 2000 repeticiones $(8,22,23,24)$.

\section{CONCLUSIÓN}

La distrofia miotónica es una enfermedad rara, por lo cual es importante divulgar sus características entre la comunidad médica para elevar el nivel de sospecha, mejorar la oportunidad en el diagnóstico, anticipar las alteraciones orgánicas, establecer pronóstico y realizar consejería genética.

\section{REFERENCIAS}

1. Kumar A, Agarwal S, Agarwal D, Phadke SR. Myotonic dystrophy type 1 (DM1): A triplet repeat expansion disorder. Gene 2013; 522(2):226-30.

2. Cassidy S, Allanson J. Management of Genetic Syndromes. 2nd Edition. New Jersey, John Wiley \& Sons, Inc; 2005.

3. Höweler CJ, Busch HF, Geraedts JP, Niermeijer MF, Staal A. Anticipation in myotonic dystrophy: fact or fiction? Brain 1989; 112 (Pt 3):779-97.

4. Prior TW. Technical standards and guidelines for myotonic dystrophy type 1 testing. Genet Med 2009; $19 ; 11(7): 552-5$.

5. Savić Pavićević D, Miladinović J, Brkušanin M, Šviković S, Djurica S, Brajušković G, et al. Molecular Genetics and Genetic Testing in Myotonic Dystrophy Type 1. Biomed Res Int 2013; 2013:1-13.

6. Mahadevan M, Tsilfidis C, Sabourin L, Shutler G, Amemiya C, Jansen G, et al. Myotonic dystrophy mutation: an unstable CTG repeat in the $3^{\prime}$ untranslated region of the gene. Science 1992; 255(5049): 1253-5.

7. GeneReviews $®$ [Internet]. Disponible en: https://www.ncbi.nlm.nih.gov/books/NBK1165. Consultado Mayo de 2016

8. Harley HG, Brook JD, Rundle SA, Crow S, Reardon W, Buckler AJ, et al. Expansion of an unstable DNA region and phenotypic variation in myotonic dystrophy. Nature 1992; 355(6360):545-6.

9. Online Mendelian Inheritance in Man, OMIM® [Internet]. Disponible en: http://omim.org/. Consultado Mayo de 2016

10. Botta A, Rinaldi F, Catalli C, Vergani L, Bonifazi E, Romeo V, et al. The CTG repeat expansion size correlates with the splicing defects observed in muscles from myotonic dystrophy type 1 patients. J Med Genet 2008; 45(10):639-46.

11. Usdin K. The biological effects of simple tandem repeats: Lessons from the repeat expansion diseases. Genome Res 2008; 18(7):1011-9.

12. Klesert TR, Cho DH, Clark JI, Maylie J, Adelman J, Snider L, et al. Mice deficient in Six5 develop cataracts: implications for myotonic dystrophy. Nat Genet 2000; 25(1):105-9. 
13. Frisch R, Singleton KR, Moses PA, González IL, Carango P, Marks HG, et al. Effect of triplet repeat expansion on chromatin structure and expression of DMPK and neighboring genes, SIX5 and DMWD, in myotonic dystrophy. Mol Genet Metab 2001; 74(1-2):281-91.

14. Chahine M, George AL. Myotonic dystrophy kinase modulates skeletal muscle but not cardiac voltagegated sodium channels. FEBS Lett 1997; 412(3):621-4.

15. Tishkoff DX, Filosi N, Gaida GM, Kolodner RD. A novel mutation avoidance mechanism dependent on S. cerevisiae RAD27 is distinct from DNA mismatch repair. Cell 1997; 88(2):253-63.

16. Mankodi A, Urbinati CR, Yuan QP, Moxley RT, Sansone V, Krym M, et al. Muscleblind localizes to nuclear foci of aberrant RNA in myotonic dystrophy types 1 and 2 . Hum Mol Genet 2001; 10(19):2165-70.

17. Miller JW, Urbinati CR, Teng-Umnuay P, Stenberg MG, Byrne BJ, Thornton CA, et al. Recruitment of human muscleblind proteins to (CUG)(n) expansions associated with myotonic dystrophy. EMBO J 2000; 19(17):4439-48.

18. Kanadia RN, Johnstone KA, Mankodi A, Lungu C, Thornton CA, Esson D, et al. A muscleblind knockout model for myotonic dystrophy. Science 2003; 302(5652):1978-80.

19. Llamusi B, Artero R. Molecular Effects of the CTG Repeats in Mutant Dystrophia Myotonica Protein Kinase Gene. Curr Genomics 2008; 9(8):509-16.

20. Timchenko NA, Cai ZJ, Welm AL, Reddy S, Ashizawa T, Timchenko LT. RNA CUG repeats sequester CUGBP1 and alter protein levels and activity of CUGBP1. J Biol Chem 2001; 276(11):7820-6.

21. Ho TH, Bundman D, Armstrong DL, Cooper TA. Transgenic mice expressing CUG-BP1 reproduce splicing mis-regulation observed in myotonic dystrophy. Hum Mol Genet 2005; 14(11):1539-47.

22. Harley HG, Rundle SA, MacMillan JC, Myring J, Brook JD, Crow S, et al. Size of the unstable CTG repeat sequence in relation to phenotype and parental transmission in myotonic dystrophy. Am J Hum Genet 1993; 52(6):1164-74.

23. Hunter A, Tsilfidis C, Mettler G, Jacob P, Mahadevan M, Surh L, et al. The correlation of age of onset with CTG trinucleotide repeat amplification in myotonic dystrophy. J Med Genet 1992; 29(11):774-9.

24. Redman JB, Fenwick RG, Fu YH, Pizzuti A, Caskey CT. Relationship between parental trinucleotide GCT repeat length and severity of myotonic dystrophy in offspring. JAMA 1993; 269(15):1960-5. 